\title{
Effects of physicochemical parameters on colloidal potentials
}

\begin{abstract}
In particle deposition problems, colloidal potentials play an important role in adsorpting the colloidal particles onto the surface of the deposit wall once the colloids arrive in the vicinity of the wall. Therefore it is important to gain understanding of these potentials, in particular, how they are influenced by the problem parameters. With such an understanding, more insights into mitigating the problem can be obtained, and consequently, more effective approaches to tackle the problem can be taken. In this work, we present the effects of particle size, flow temperature and ionic strength of the solution on the colloidal potentials based on numerical analysis. The results support the conclusion that the rate of initial deposition of particles can be reduced if the particle size is smaller, the flow temperature is increased, and the ionic strength is reduced.
\end{abstract}

Keyword: Colloidal potentials; DLVO theory; Particle deposition 